\title{
Muscular Movements of Fishes*
}

\section{By Dr. J. Gray, F.R.s.}

$\mathrm{I}^{\mathrm{P}}$ a body is moving through water at a constant speed, the body must be constantly supplied with energy. In the case of a fish, the requisite energy is supplied by the muscles and is applied in such a way as to exert a backward thrust on the water, which compensates the resistance exerted by the water on the body of the moving fish.

As observed by the human eye, the motions of various types of fish appear to vary considerably from one species to another. At one extreme is the eel, which, during motion, is characterised by distinct waves of curvature which pass alternately down each side of the body from head to tail. At the other extreme is the mackerel or the trout, which appears to progress by means of transverse strokes of the expanded caudal fin. An examina. tion of successive instantaneous photographs shows, however, that the nature of these two types of movement is essentially the same, for in all cases, waves of curvature pass along the body with increasing amplitude as the hind end of the fish is approached. The only significant differences between the eel and the mackerel are the relatively larger amplitude of the waves towards the anterior end of the eel, and the relatively longer bodywith consequently longer length of wave-in the eel as compared with the mackerel. Both the nature and frequency of the waves of muscular curvature vary in different cases.

As recorded by the eye, the movements of a fish are almost invariably movements of one part of the body relative to another-we watch the waves of curvature passing backwards relative to the head of the eel, or we watch the tail of a mackerel sweeping from one side of the body to the other. From a propulsive point of view, however, we are more concerned with the motion of the body relative to the surrounding water, and in order to record such movements it is necessary to employ a camera, and to photograph the fish, against a elearly defined background, at known intervals of time. Observations of this type enable us to record the movements of each part of the body relative to other parts and relative to the surrounding medium.

Records have been made of a number of typical fish, but, owing to the well-defined nature of the muscular waves, attention may be first concentrated on a small glass eel (Anguilla vulgaris) which is approximately $7 \mathrm{~cm}$. in length. It is found that each point on the animal's body is travelling forward through the water along a sinusoidal curve the 'pitch' or wave-length of which is the same for all points of the body (namely, $3.2 \mathrm{~cm}$.) but the transverse amplitude of which becomes progressively greater as we select points lying farther away from the head. If we

* Friday evening discourse before the Royal Institution delivered on April 28. select two adjacent points on the body and plot out their movements relative to the background of the fish, it is seen that each sector of the body is moving with its leading surface inclined at an angle to its own path of motion. The photographic records also show that, relative to the head of the fish, each leading surface is always directed obliquely backwards except at the extreme positions of the displacement.

The leading surface of the fish has therefore two distinct properties: (1) it faces obliquely backwards relative to the head of the fish ; and (2) it is moving at an angle to its own direction of motion. These two properties are responsible for the forward propulsion of the fish. So long as the leading surface is moving at an angle with its own path of motion, there will be a pressure exerted at right angles to the surface, and so long as the leading surface is directed obliquely backwards relative to the head of the fish, the pressure will be directed obliquely forwards. The forward component of this pressure tends to drive the fish forwards.

The principle is fundamentally the same as in a screw-propeller or in the blade of a sculling oar during the first part of its stroke. In the screw propeller the leading surfaces of the blades represent surfaces directed obliquely backwards relative to the ship and, when the ship is in motion, they are moving forward at an angle to their own path of motion. In the case of the fish, the propellant surfaces are moving in two dimensions instead of in three-and this is possible by virtue of the elasticity of the body, which enables the leading surface to be suitably reorientated at the end of each transverse cycle.

The surface of a screw and the leading surface of a fish's body resemble each other in that they are always exerting a pressure at right-angles to themselves, although, in the fish, the extent of this pressure varies at different points on the body and at different phases of each transverse movement. The waves which pass down the body of a fish also have their counterpart in the mechanical screw. If the pointed end of a household screw is held vertically downwards and the screw be rotated, it can be seen that the edges of the screw surface appear to move along the side of the screw in a direction which varies with the direction of rotation. If the screw is turned 'in' the ridges appear to move upwards; if the screw is turned 'out' the ridges appear to move downwards. If, however, the screw is free to move in a well-fitted nut, the ridges appear to be stationary when the screw is rotated in either direction. It can be shown fairly simply that the screw will only do work when the 'ridges' appear to move upwards or downwards at a greater rate than that at which the screw itself is moving in an opposite direction. The same facts can be summarised by saying that the screw will only exert a pressure when there is 
an 'angle of attack' between its leading surface and the direction of the latter's motion; within limits, the greater is this angle of attack the greater is the pressure exerted by the screw. So also in the eel, the surface of the body can only exert a forward thrust when the waves of muscular contraction pass backwards along the body at a greater rate than that at which the fish is moving forwards through the water. When a wave passes down the body of a fish at rest, the angle of attack is, at first, of maximum value and the thrust is correspondingly high-consequently the fish rapidly gathers speed until the angle of attack gives a thrust which is equal to the forces resisting the motion of the whole fish; during this period, there has been a steady reduction in the rate at which the muscular waves are travelling backwards relative to the environment. If the fish, having acquired a uniform speed, increases the velocity at which it transmits the waves along its body, then the angle of attack will again rise. A second period of acceleration of movement will occur until the angle of attack has again fallen to an equilibrium value. For any given speed of transmission of the waves along the body there must be a definite speed of forward propulsion which depends on the form of the fish's body. A series of waves passing down the body of a fish must therefore automatically travel at a greater speed than that at which they propel the fish forwards. Correspondingly, if the direction of the waves is reversed, the fish moves backwards-a phenomenon clearly seen in a large conger eel.

We may conclude that the whole body of the eel is acting as a propeller by virtue of the fact that the leading surface is always inclined obliquely backwards relative to the head and at an angle to its own path of motion.

If we now consider the movements of a whiting or other typically pelagic fish, certain points of difference are obvious : (1) the length of the body, relative to its cross-section, is much less than in the case of the eel ; (2) the hind end of the body tapers relatively abruptly and ends in an expanded caudal fin. When recorded photographically, however, the movements of the body are found to be of the same fundamental type as those of the eel, for in all cases waves of curvature pass alternately down each side of the fish.

In the whiting, the amplitude of these waves remains quite small until the posterior region of the tail is reached, where it increases more abruptly than in the case of the eel. The effect of these waves is such as to maintain the leading surface of the body inclined obliquely backwards relative to the head. A similar orientation is maintained by the leading surface of the caudal fin, and since the area of the fin is considerable, it is of interest to consider how far it plays an essential rôle as a propellant surface. It is well known that fish with badly lacerated caudal fins can swim actively and the observations of Breder show that amputation of the caudal fin of Scardineus does not markedly affect the speed of propulsion. This observation has been confirmed by the removal of the caudal fins of the rudd, the perch, and the whiting. For technical reasons it has not been possible to observe the effect of amputation of the caudal fin in fish moving at a high speed, but it seems fairly clear that the 'cruising' speed is not appreciably reduced.

At first sight, these facts suggest that the main propulsive surface of a whiting is provided by the body itself and that the surface of the caudal fin is relatively insignificant. An examination of the photographic records, however, shows that the removal of the caudal fin alters, in a significant manner, the movements of the fish. In the intact fish, we have seen that the leading surface of the body is always directed obliquely backwards. When the caudal fin has been removed, however, this is no longer the case, for the stump of the tail now swings about its base as a relatively rigid structure; as it moves from the extreme position of displacement towards the central position its leading surface is directed obliquely backwards, but as it moves from the central position towards the extreme position of displacement on the other side, the leading surface is directed obliquely forwards.

The difference between the movements of the intact fish and those of one from which the caudal fin has been removed is clearly due to the resistance which the tail fin offers to transverse movement. The fin represents a surface of high resistance lying posteriorly to the region of muscular contraction and it causes the intervening region of the body (which is flexible) to bend into an arc, with the convex surface towards the direction of motion; in other words, the fin causes the posterior region of the body to lag behind all those parts which lie between itself and the point of contraction so that each group of muscles, as it comes into play, is operating on a region of the body which is directed obliquely backwards relative to the head. The effect of the fin is exactly parallel to that produced by attaching a flat plate to the distal end of a steel wire and oscillating the proximal end of the wire through a small angle. Without a flat plate each part of the wire moves in practically the same phase as any other part-but when the plate is present the distal end of the wire lags behind the proximal end. A series of movements is set up which is strikingly similar to the normal movements of a fish's body; without the attached plate, the movements of the wire are comparable to those of a fish from which the tail fin has been removed.

At first sight, it seems strange that a fish can move forwards without a tail fin, for one might imagine that the propulsive effect produced when the tail is directed obliquely backwards would be exactly neutralised by the action of the tail when directed obliquely forwards during the second half of each stroke. It must be remembered, however, that so far we have been considering the movements of the tail relative to the head. When we 
examine the movements of the tail relative to the water, we see how the fish-without a caudal fincan propel itself through the water. During the first half of the stroke, the tail is not only directed obliquely backwards but it also sweeps through the water, thereby generating a forward thrust, but at the moment it begins to face obliquely forwards, its motion through the water is checked, for it begins to move against the water which is flowing past the sides of the fish. The tail then begins to act as a rudder, and the position of the tail relative to the head is maintained by a movement of the head end of the fish through the water instead of by a movement of the tail. The two phases of each stroke are thus asymmetrical and the fish is able to progress through the water, although not in the smooth steady manner characteristic of the intact fish.

It is clear that by exerting a mechanical resistance to transverse movement through the water, the caudal fin of the whiting maintains the necessary phase difference between successive regions of the muscular body and tail, and thereby enables the whole of the energy of the muscular contractions to be expended in a smooth symmetrical manner. The question may be asked: Why can an eel or dogfish progress smoothly without the presence of a large caudal fin? The answer is that the function of the caudal fin of a whiting is, in the case of the eel or dogfish, carried out by means of the long flexible body itself. In exactly the same way, well-defined waves can be transmitted along a long flexible wire without the presence of a flat plate attached to the distal end. It is only when the wire is short that a flat plate is essential for maintaining an adequate phase difference between successive regions along its length.

Although the removal of the caudal fin does not substantially reduce the forward rate of movement, it is not legitimate to assume that the propulsive thrust from the fin is negligible in the intact animal, for it has been shown that amputation of the fin induces far-reaching changes in the movements of the remainder of the body relative to the surrounding water. A preliminary attempt to estimate the propulsive effect of the tail fin of the whiting was carried out as follows.

A series of vertical rods was so mounted in a metal frame that each rod moved harmonically with the amplitude characteristic of different levels of a whiting's body; the ends of the rods were then inserted into the body of a dead whiting. By rotating a handle, the body of the fish was forced to transmit a series of waves identical with those seen in the normal living fish, and for a given frequency of waves the velocity of the current produced in a tank of water was determined. The caudal fin was then removed and the current again measured for the same frequency of movement. Although the method of measuring the current was notfree from criticism, theresults suggest that approximately 40 per cent of the propulsive thrust from a whiting is derived from the caudal fin.
It is of interest to note that just as the pro. pulsive thrust represents the forward component of the pressure of the fin against the water, so the transverse component of this pressure plays its part in adding to the resistance offered by the fin to transverse movements, so that the biological functions of the fin are very clearly linked together, just as is the case with the surface of the body itself. If the area of the body surface is large in comparison to that of the fin, the rôle of the fin may be relatively insignificant as in the eel, but in the pelagic type of fish the posterior region of the muscular tail is comparatively narrow and tends to present a convex surface to the water, whereas the tail fin is relatively large and presents a flat surface to the water. In the latter case the fin probably plays an increasingly important rôle as a propeller, just as it plays an increasingly important role by virtue of its resistance. Until a very accurate method is devised for measuring the currents produced by various types of tail fin, it is dangerous to speculate on the effect produced by variations in the shape of the caudal fin.

So far, we have only considered the motion of fish which are swimming in a straight line, but one of the most striking features of a moving fish is its ability to change the direction of its motion. In some cases such changes are effected by movements of the paired fins, but the rapid changes so characteristic of pelagic types are effected by the muscles of the body itself. In all cases a change in the direction of motion has been found to be due to the propagation of a muscular wave along one side of the body, and the fish always turns towards the side along which the wave is travelling. These waves are most conspicuous in the case of the eel, but they can be recorded photographically in other types. We may take as an extreme example the goldfish. As observed by the eye, a goldfish appears to turn by a rapid flexure of the tail towards one side. Actually, however, the tail remains practically stationary in the water until the head end of the fish has almost if not completely finished its turning movement-after which the tail swings through the water towards the opposite side to which the animal turns.

The examination of a number of photographic records has shown that the turning movement of such fish is divisible into two parts: (1) the head moves through the water by using the tail fin as a relatively stationary pivot; (2) the tail moves through the water using the head as a stationary pivot. The effect of the tail fin during the first phase of the movement is simply an exaggeration of its effect during normal swimming, namely, to delay the movements of the hind end of the body whilst movements are occurring farther towards the head. As one might expect, the amputation of the caudal fin exerts a very farreaching effect on the turning powers of the fish. A normal goldfish can turn through nearly $90^{\circ}$ by one flexure of the body-but if the caudal fin be removed, the same turn can only be effected by several flexures. With or without a caudal fin 
the amplitude of the muscular contractions is practically the same, but in the absence of the caudal fin a muscular contraction on the left side of the body causes both head and tail to move simultaneously to the left of the original axis-in the presence of a caudal fin only the head moves whereas the tail remains stationary. Since the curvature of the body is the same in the two cases, it follows that the angular displacement of the head through the water is greatly reduced by the amputation of the fin. The maximum change in the direction of the fish cannot exceed the initial movement of the head, and this can only be maximal when the posterior end of the body remains undisplaced. Clearly the function of the tail fin is to enable the hind end of the body to act as a fulcrum on which the head turns. As in normal motion, the role of the caudal fin is, in the eel or dogfish, carried out by the long flexible body itself.

We have now seen that the caudal fin exerts two forces on a moving fish: (I) it tends to inhibit the transverse movements of the hind end of the body ; (2) it exerts a fraction of the forward propulsive thrust. Both these forces are derived from the pressure exerted on the water at right angles to the surface of the fin. 'The 'resistant' force is the transverse component of this pressure, and the propulsive thrust is the forward component. The absolute and relative values of these forces remain to be determined.

So far, we have considered the movements of the body without reference to the actual process of contraction of the muscles. The muscle fibres of the trunk and tail all run parallel to the long axis of the body, and they are so controlled that when a fibre on one side of the body is fully contracted, its opposite neighbour on the other side of the body is fully relaxed. Further, it is clear that the fibres along one side of the body do not contract simultaneously but in sequence, beginning with those nearest to the anterior end of the body. Each group of fibres is supplied by motor nerves, and it has been suggested that a muscular wave is the result of the passage of a nervous disturbance down one side of the spinal cord, firing off each group of fibres as it goes. It is difficult, on this view, to account for the very variable speed of transmission of the muscular waves.

There are, on the other hand, a number of facts which suggest that when the muscles (lying on one side of the base of the muscular tail) contract, their energy can be transmitted mechanically along the body of the fish just as such energy can be transmitted along the length of an elastic wire. The energy is transmitted in the form of tension by the stretched skin and muscles of the leading side of the body. If the muscles lying nearer to the tip of the tail are to be usefully employed, they must liberate their energy in phase with that of the mechanical wave. When the mechanical wave reaches any given point it first tends to store energy at that point by bending the body into a convex curve -and then this energy is afterwards liberated as the tension is released. If, therefore, the muscles at any point are to supplement this mechanical cycle, it follows that they must begin to contract at the moment when a similar shortening process is being induced by the mechanical wave. This would occur if the stimulus to contract were automatically induced by a stretching of the muscle itself. Such a proprioceptive mechanism is well defined among other vertebrate types and its existence in fish would account for many otherwise unrelated facts.

\section{Low Auroras}

$\mathrm{I}^{\mathrm{T}}$ is frequently asserted (a) that the aurora occasionally descends to the earth's surface so that it can be seen between the observer and relatively near terrestrial objects, and $(b)$ that not infrequently unmistakable sounds accompany auroral displays. Several writers have collected reports from residents in regions where the aurora occurs and a large mass of evidence for the reality of low auroras and sounds accompanying auroral display has been accumulated. In NATURE for March 7, 1931, Prof. S. Chapman reviewed one such collection of reports and expressed his opinion that "These letters make it difficult to deny that auroræ occur, very rarely, quite near the earth, and are sometimes accompanied by noises".

In an article which has just been published in the Quarterly Journal of the Royal Meteorological Society (vol. 59, p. 249), Dr. G. C. Simpson discusses the whole problem of low auroras and auroral sounds and comes to the conclusion that "the evidence for low auroras is extremely weak and that when one weighs that evidence against the positive information we have about the nature and position of the aurora one is justified in denying that auroras have ever been observed below a height of $80 \mathrm{~km}$." With regard to sounds, Dr. Simpson says that he is not prepared to deny the possibility that there may be some form of objective sound associated with the aurora, but he very much doubts it.

Dr. Simpson uses three main arguments against the reality of low auroras. In the first argument he points out that we now know that the chief spectroscopic line of the light of the aurora is due to a metastable state of atomic oxygen. This condition of oxygen is only possible when the pressure is very low, and trigonometrical measurements have proved that the height of the ordinary aurora is more than $80 \mathrm{~km}$., where the pressure is sufficiently low. If now an auroral streamer passes from this height to the earth's surface where the pressure is too high for atomic oxygen to exist, a second physical process must come into action, and this process, whatever it may be, would have to come into action in exactly the same pro. portion as the effect of the first process decreased, 